\title{
Estratigrafía de 'sin-rift' (Triásico Superior-Jurásico Inferior) de la Cuenca Neuquina en la sierra de Chacaico, Neuquén, Argentina
}

\author{
Juan R. Franzese \\ Gonzalo D. Veiga \\ Martín Muravchik
}

María Daniela Ancheta

Leandro D'Elía

\author{
Universidad Nacional de La Plata (UNLP), Calle 1 No. 644, B1900 TAC, La Plata \\ CONICET, Avda. Rivadavia 1917-CP C1033 AAJ, Argentina \\ franzese@cig.museo.unlp.edu.ar \\ veiga@cig.museo.unlp.edu.ar \\ mura@cig.museo.unlp.edu.ar \\ Universidad Nacional de La Plata (UNLP), Calle 1 No. 644, B1900 TAC, La Plata \\ daniela@cig.museo.unlp.edu.ar \\ Universidad Nacional de La Plata (UNLP), Calle 1 No. 644, B1900 TAC, La Plata \\ CONICET, Avda. Rivadavia 1917-CP C1033 AAJ, Argentina \\ Idelia@cig.museo.unlp.edu.ar
}

RESUMEN

La sierra de Chacaico (Neuquén, Argentina) constituye una de las localidades más importantes para el análisis de la estratigrafía y relaciones espaciales del relleno inicial de la Cuenca Neuquina. Por medio de estudios estructurales y sedimentológicos de campo ha sido posible distinguir la naturaleza volcano-sedimentaria de la secuencia de 'sin-rift' así como su evolución estratigráfica durante el período extensional Triásico Superior-Jurásico Inferior. La sucesión de 'sin-rift' puede subdividirse en tres etapas evolutivas. La parte inicial está integrada por volcanitas básicas a intermedias y depósitos sedimentarios volcanoclásticos, mayormente gruesos. La sección media se halla dominada por depósitos piroclásticos primarios y reelaborados y finaliza con delgadas secuencias carbonáticas lacustres. En el techo de esa sección aparecen localmente lavas basálticas. La sección superior consiste de una asociación de depósitos clásticos gruesos (conglomerados y brechas) y piroclásticos primarios de caída y de flujo. Toda el área estudiada conformó un único depocentro de 'sin-rift' cuyos márgenes estaban orientados en dirección E-W a ENE-WSW. El borde sur funcionó como la estructura de fallamiento normal más importante, hacia la cual los espesores de la secuencia fueron máximos. El depocentro fue invertido tectónicamente de manera compleja, con generación de anticlinales asociados a fallas normales invertidas. Algunas de esas estructuras anticlinales reflejan la existencia de escalones estructurales internos dentro del depocentro, asociados a antiguas fallas normales oblicuas de orientación noreste. El patrón estratigráfico y estructural de la secuencia de 'sin-rift' es comparable al de la dorsal de Huincul, uno de los rasgos morfoestructurales más prominentes en la evolución del sector sur de la Cuenca Neuquina.

Palabras claves: Cuenca Neuquina, 'Rift', Estratigrafía, Triásico-Jurásico, Sedimentación volcanoclástica, Argentina.

\section{ABSTRACT}

Syn-rift stratigraphy (Upper Triassic-Lower Jurassic) of the Neuquén Basin at the Sierra de Chacaico, Neuquén, Argentina. The sierra de Chacaico (Neuquén, Argentina) is one of the most important localities to study the Upper Triassic-Lower Jurassic syn-rift sequence of the Neuquén Basin. Structural and sedimentological analyses have been carried out in order to depict the stratigraphic evolution of the infill. The syn-rift sequence evolved in a volcano- 
sedimentary environment. For the entire column, three sections have been recognized. The lowest section is composed of basic to intermediate volcanic rocks associated with coarse-grained volcaniclastic deposits. The middle section is dominated by pyroclastic flow and fall deposits closely associated with volcaniclastic sandstones and minor conglomerates. Lacustrine carbonates and basaltic lavas were recognized at the top of the middle section. The upper section is characterized by coarse-grained conglomerates and breccias and pyroclastic fall and flow deposits. The whole area formed a unique depocentre during syn-rift evolution, its margins trending E-W/ENE-WSW. The southern margin acted as the main active border of the trough, where the thickest section was deposited. The syn-rift depocentre was inverted during later tectonic events leading to the generation of a complex pattern of anticlines and reverse faults. Some of these anticlines were formed by the inversion of oblique trending (north-east) normal faults suggesting the presence of internal steps within the depocentre. The structural and stratigraphic pattern observed at sierra de Chacaico is similar to the main framework of the Huincul high, one of the most prominent tectonic elements of the southern Neuquén Basin.

Key words: Neuquén Basin, Rift, Stratigraphy, Triassic-Jurassic, Volcaniclastic sedimentation, Argentina.

\section{INTRODUCCIÓN}

La Cuenca Neuquina constituye uno de los sistemas de acumulación de sedimentitas mesozoicas más importante de los Andes centrales argentino-chilenos. La cuenca se inició como consecuencia del colapso extensional de un orógeno de edad paleozoica superior. Posteriormente su evolución fue controlada por el crecimiento del arco magmático andino y una tectónica multiepisódica que provocó fenómenos de inversión local en diversas áreas de la cuenca desde el Jurásico (Legarreta y Uliana, 1996; Vergani et al.,1995; Franzese y Spalletti, 2001).

La cuenca registra al menos dos episodios de 'rift'. La primera etapa ('rift' inicial), de edad triásica superior a jurásica inferior, produjo un complejo conjunto de cubetas alargadas y desconectadas entre sí que evolucionaron mayormente en un ambiente continental volcánico (Fig. 1). La segunda etapa de 'rift' ocurrió durante el Jurásico Inferior (Pliensbachiano-Toarciano) en un ambiente mayormente marino en áreas más localizadas de la cuenca (Vergani et al., 1995).

El relleno de 'sin-rift' del primer evento extensional ('rift' inicial) es conocido a la escala de la cuenca como Ciclo Precuyano (Gulisano et al., 1984). Esta unidad incluye las secuencias volcanosedimentarias del Triásico Superior y Jurásico Inferior aflorantes en el frente andino desde el sur de la provincia de Mendoza hasta el sur de la provincia del Neuquén. Las edades de este relleno se conocen a partir de análisis radiométricos realizados sobre ignimbritas del subsuelo, que han proporcionado valores de 219 Ma y 182 Ma

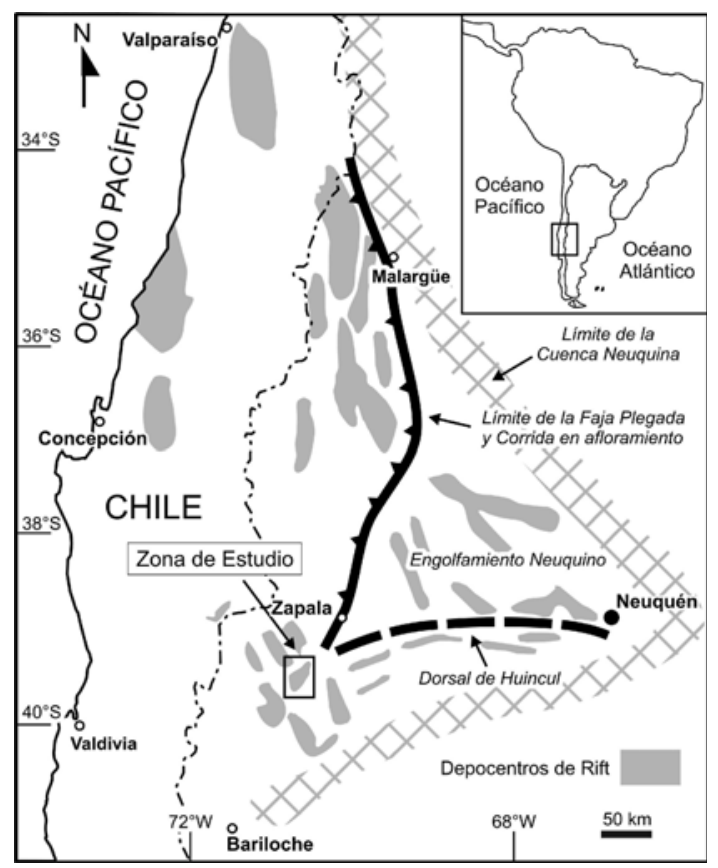

FIG. 1. Ubicación y límites de la Cuenca Neuquina y distribución de los principales depocentros de 'sin-rift' del Triásico Superior-Jurásico Inferior (modificado de Vergani et al., 1995 y Franzese et al., 2006). La dorsal de Huincul y la faja plegada y corrida señalan dos unidades morfoestructurales relacionadas con la inversión de la cuenca.

(Pángaro et al., 2002). Restos de plantas fósiles encontradas en superficie han sido considerados como de edad triásica superior (Spalletti et al., 1991). 
La estratigrafía de 'sin-rift' del evento inicial, entendida como la organización espacial y temporal del relleno asociado a la primera etapa de fallamiento normal, ha sido estudiada a grandes rasgos a partir del análisis de afloramientos de secuencias exhumadas por inversión tectónica y de trabajos de subsuelo. En los últimos años su conocimiento se ha visto incrementado debido a que integra un sistema petrolero que se halla en plena etapa de exploración. No existe aún un esquema estratigráfico refinado que integre la distribución de unidades internas a la escala de cada depocentro extensional individual, aun cuando es conocida la estratigrafía de detalle de secuencias de 'rift' del Triásico Superior-Jurásico Inferior en áreas de la Cordillera Principal, adyacentes a la Cuenca Neuquina (Álvarez y Ramos, 1999) o en otras localidades de la misma cuenca (Franzese et al., 2006). Algunas áreas de afloramiento de las secuencias de 'rift' inicial de la provincia del Neuquén poseen características ideales para un estudio de esa naturaleza, tal como la región conocida como sierra de Chacaico, ubicada en el área centro-occidental de la cuenca (Figs. 1 y 2). En esa región aparecen secciones muy completas de la secuencia estratigráfica inicial y sus relaciones con el basamento y con las secuencias que la cubren.

El propósito de este trabajo es presentar un análisis estratigráfico de las secuencias de 'sinrift' más antiguas de la Cuenca Neuquina (Triásico Superior-Jurásico Inferior) en la sierra de Chacaico, comprender la variabilidad litológica vertical y areal del relleno, establecer su relación dentro de la historia de la cuenca y ofrecer un panorama estratigráfico funcional del Precuyano a la escala de un depocentro individual. Con ese objetivo se realizaron tareas de mapeo geológico y estructural sobre la base de fotografías aéreas, imágenes LANDSAT, datos altimétricos provenientes de la carta geológica 1:200.000 y relevamientos de campo. En localidades consideradas críticas se relevaron ocho columnas estratigráficas, las cuales son analizadas y correlacionadas. Los resultados obtenidos son comparados con la historia geológica de las áreas vecinas de la cuenca.

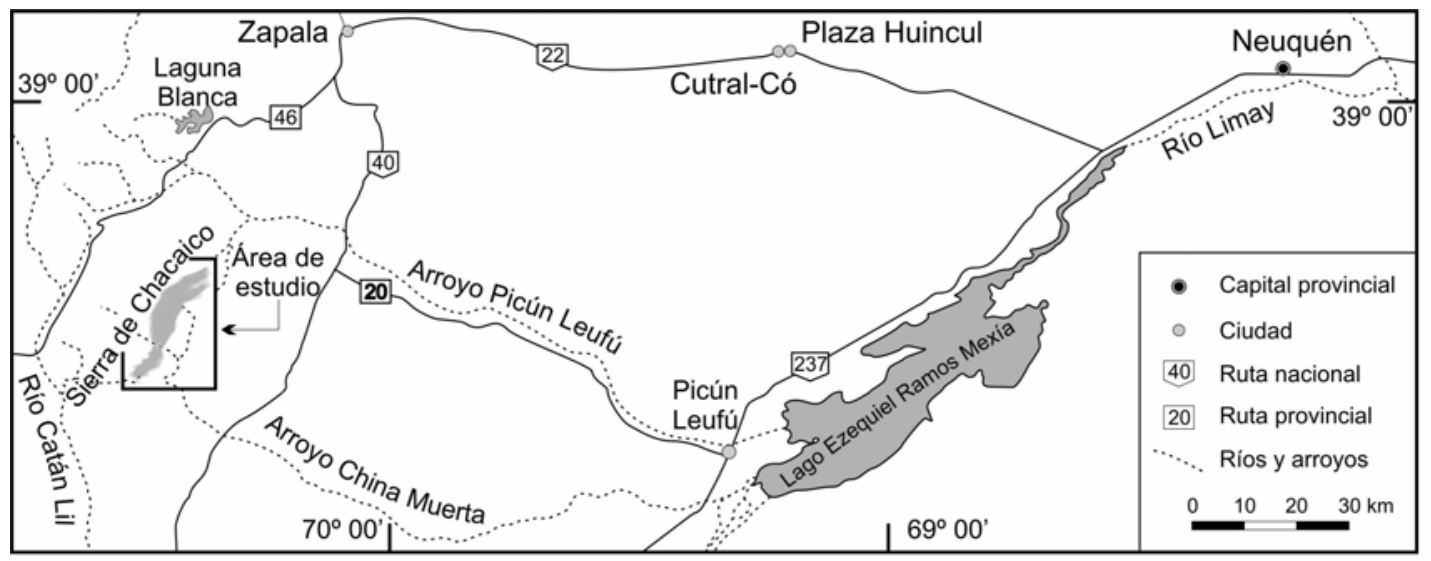

FIG. 2. Mapa de ubicación de la zona de estudio.

\section{MARCOGEOLÓGICO}

La sierra de Chacaico (Figs. 2 y 3) es un rasgo montañoso alargado en dirección NNE, relativamente oblicuo a la cadena andina actual, que se

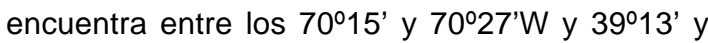
$39^{\circ} 25^{\prime}$ S. Se sitúa en un ambiente tectónico muy particular, ya que se encuentra en el área invertida por la tectónica cenozoica del arco andino y a la vez constituye la prolongación occidental de la dorsal de Huincul (Orchuela et al., 1981), estructura de inversión jurásica este-oeste más prominente de 


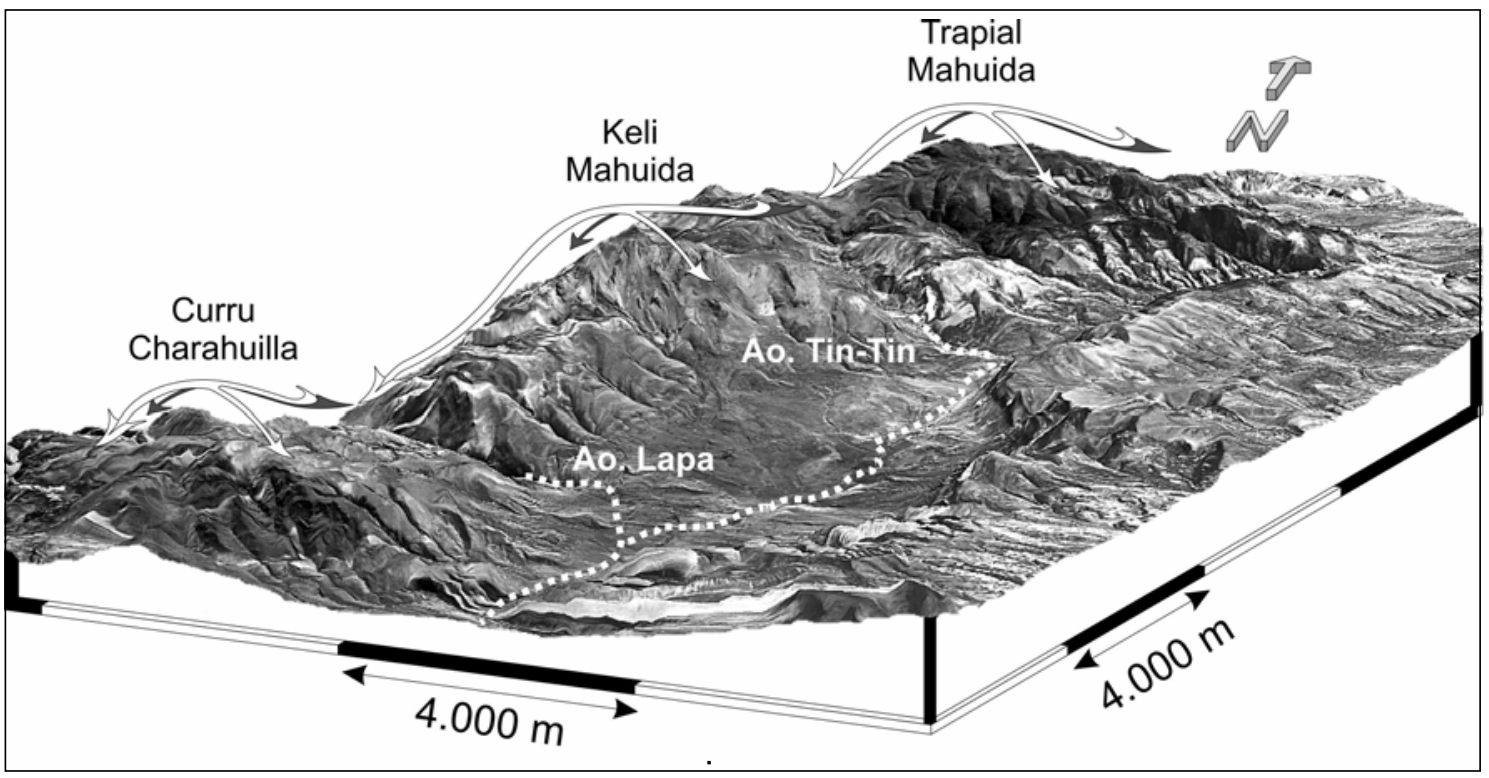

FIG. 3. Modelo de elevación digital (DEM) de la sierra de Chacaico (exageración vertical 2:1) con imagen LANDSAT TM superpuesta. Nótese las tres culminaciones anticlinales referidas en el texto.

la Cuenca Neuquina (Fig. 1). A lo largo de toda su extensión se registran afloramientos de las secuencias más antiguas de la cuenca (Lambert, 1946; Suero, 1951; Volkheimer, 1973; Leanza, 1990). El basamento está representado por esquistos y filitas de la Formación Piedra Santa, de edad devónica a carbonífera superior (Franzese, 1995), la cual se halla intruida por plutones del denominado Complejo Plutónico del Chachil (Leanza, 1990), de edad carbonífera superior a pérmica inferior (Fig. 4).

Los depósitos de 'sin-rift' en la sierra de Chacaico están integrados por rocas volcánicas efusivas, rocas piroclásticas primarias y sedimentitas epiclásticas con una alta proporción de material volcánico en su constitución. Estas unidades integran la Formación Lapa. Algunos autores han separado la parte inferior del relleno de 'sin-rift' con los nombres de Formación Choiyoi (Leanza, 1990) o Formación Ñireco (Leanza et al., 2005). En este trabajo se considera conveniente no realizar esta subdivisión en virtud de la fuerte vinculación genética observada en todo el relleno volcanoclástico continental triásico superiorjurásico inferior (Franzese y Spalletti, 2001; Franzese et al., 2006).

Los depósitos que cubren la sucesión de 'sinrift' se apoyan también sobre el basamento ígneo-
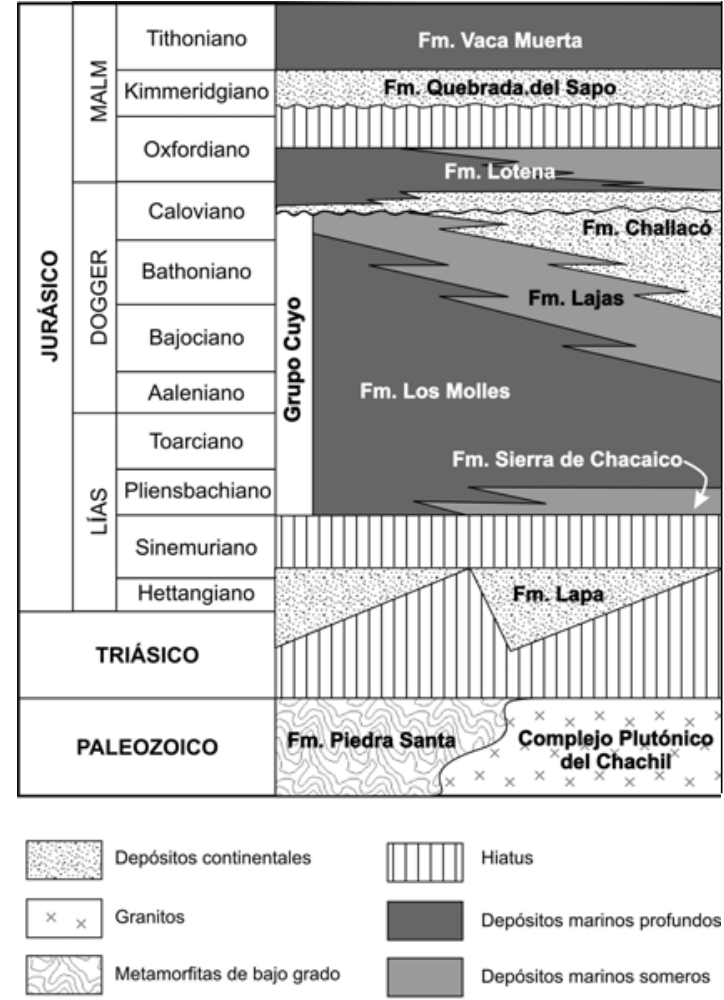

FIG. 4. Cuadro cronoestratigráfico pre-cretácico para el sector sur de la Cuenca Neuquina. 
metamórfico y constituyen, en esta localidad, el inicio del 'post-rift'. Se trata de secuencias marinas someras de edad pliensbachiana inferior designadas Formación Sierra de Chacaico (Volkheimer, 1973). Cabe señalar que no existe en el área de estudio ninguna evidencia de la segunda fase de 'rift' de edad pliensbachiana-toarciana (Vergani et al., 1995) presente en otros sectores de la cuenca. La Formación Sierra de Chacaico pasa transicionalmente a turbiditas de la Formación Los Molles (Pliensbachiano-Bajociano en el área de estudio), integrando ambas unidades la parte basal del Grupo Cuyo. Por encima de estas secuencias se apoya una espesa pila sedimentaria, de más de $2.000 \mathrm{~m}$, que incluye unidades continentales y marinas que alcanzan el Cretácico Inferior (Fig. 4).

\section{ESTRUCTURA DE LA SIERRA DE CHACAICO}

La observación de los rasgos geológicos y geomorfológicos de campo indica que la sierra de Chacaico es una estructura anticlinal con eje orientado en dirección NNE y vergencia hacia el oeste (Figs. 3 y 5). El flanco oriental posee inclinaciones promedio de $10^{\circ}-15^{\circ} \mathrm{E}$ mientras que el occidental, asociado parcialmente a una falla inversa que no llega a emerger totalmente a lo largo de la sierra, está más inclinado $\left(40^{\circ}-50^{\circ} \mathrm{W}\right)$ y en algunos sectores llega a disponerse verticalmente. El eje del anticlinal está curvado hacia el este en su extremo norte y hacia el oeste en su extremo sur (Fig. 5). En ambos extremos la sierra de Chacaico termina abruptamente: en su límite norte finaliza contra una importante falla de orientación este-oeste que levanta el basamento por sobre niveles del 'post-rift' (Grupo Cuyo) mientras que en su límite sur se intercepta contra una falla de la misma orientación (Falla de Las Coloradas, Fig. 5) que presenta su labio norte levantado con un fuerte rechazo que se podría estimar en el orden de los $1.000 \mathrm{~m}$.

Si bien el anticlinal que conforma la sierra de Chacaico muestra una orientación preferencial NNE, es notoria la existencia de estructuras de plegamiento transversales y oblicuas al eje principal de la sierra, algunas de ellas subparalelas a la disposición de los abruptos límites norte y sur (Fig. 5). Esas estructuras constituyen el rasgo de mayor magnitud de la sierra y provocan la generación de tres culminaciones en el anticlinal de Chacaico en las cuales han sido sobreelevados estructuralmente elementos estratigráficos tanto del 'pre-rift' como del 'sin-rift'. La culminación que se halla ubicada al norte del área de estudio (cerro Trapial Mahuida, Figs. 3 y 5) está marcada por un bloque de basamento fallado e inclinado al sureste. La continuación de ese bloque hacia el suroeste engrana con una antiforma de orientación noreste en cuyo núcleo asoman rocas del 'sin-rift' (Fig. 5).

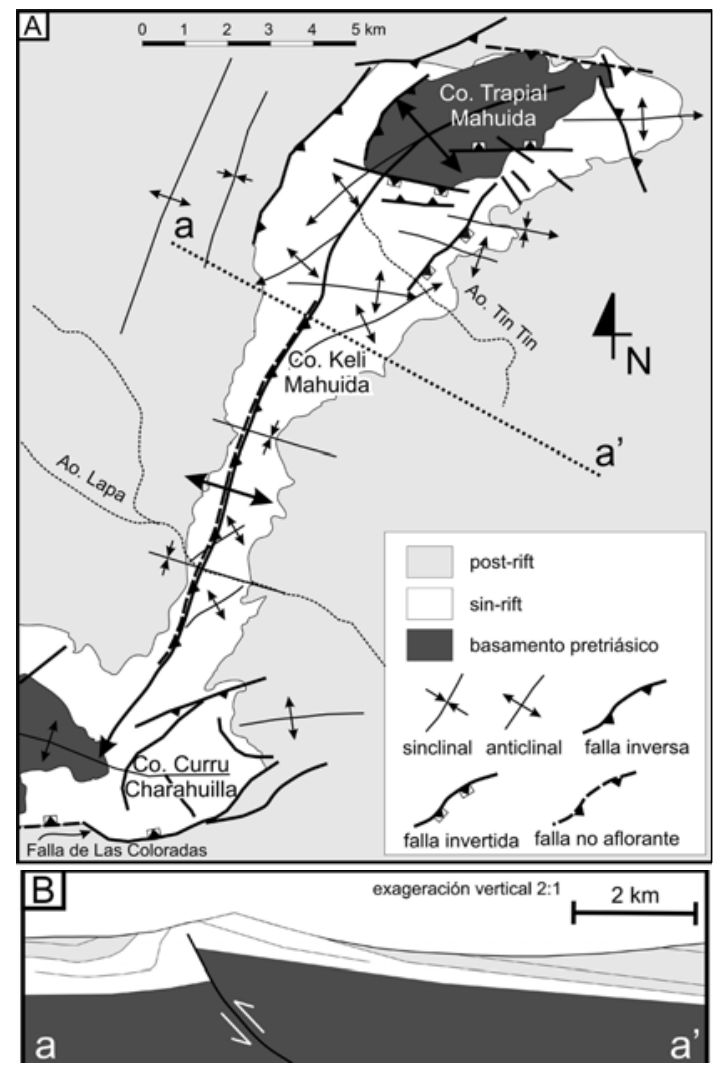

FIG. 5. A. Mapa estructural de la sierra de Chacaico. Las fallas invertidas corresponden a estructuras extensionales directamente relacionadas con la depositación de las secuencias de 'sin-rift', reactivadas en forma inversa. $\mathbf{B}$. Corte estructural. Nótese la vergencia de las estructuras hacia el oeste, así como la diferencia de inclinación de los limbos del pliegue.

Más al sur, en la zona central de la sierra, otra estructura anticlinal oblicua con disposición noreste levanta depósitos de 'sin-rift' que forman el cerro Keli Mahuida (Figs. 3 y 5). Finalmente, el extremo 
sur del anticlinal conforma una tercera culminación, de forma dómica, vinculada con la intersección del anticlinal de Chacaico y otro anticlinal con eje este- oeste subparalelo a la Falla de Las Coloradas, que pone límite a toda la estructura (Fig. 5).

\section{ESTRATIGRAFIA DE 'SIN-RIFT'}

De acuerdo a las observaciones de campo y al levantamiento de ocho secciones estratigráficas distribuidas a lo largo de la sierra, el relleno de 'sinrift' puede ser subdividido en tres unidades infor- males, caracterizadas por rasgos litológicos particulares y distribución espacial fuertemente relacionada con la geometría de la sierra y con la disposición de las unidades de 'pre-rift' (Figs. 6 y 7).

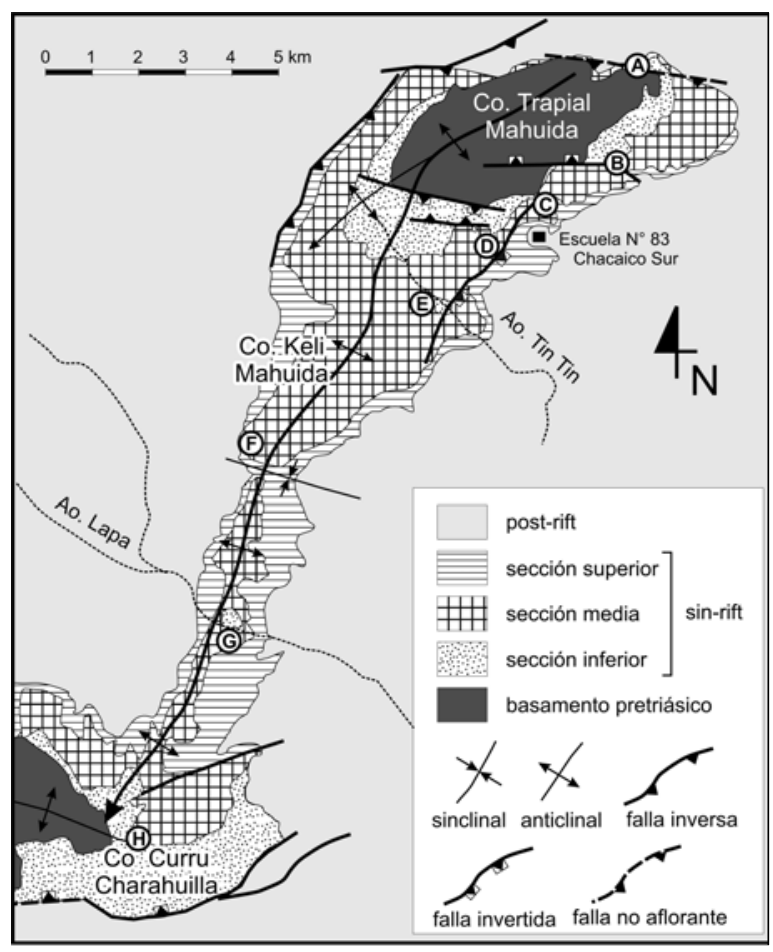

FIG. 6. Mapa geológico de la sierra de Chacaico mostrando la distribución de las unidades de 'sin-rift', su relación con las principales estructuras y las rocas de 'pre-' y 'post-rift' y la localización de las secciones de detalle estudiadas.

\section{SECCIÓN INFERIOR}

La unidad inferior de la secuencia de 'sin-rift', con potencias de más de $300 \mathrm{~m}$, está compuesta por rocas volcánicas lávicas básicas y mesosilícicas asociadas con depósitos volcánoclásticos de tonalidades moradas y grises. Esta sección muestra ciertas diferencias composicionales entre las distintas localidades estudiadas. En el área norte se observan lavas andesíticas $\mathrm{y}$, en menor proporción, basálticas, frecuentemente autobrechadas, con potencias individuales de hasta $10 \mathrm{~m}$, intercaladas con depósitos volcanoclásticos, mayormente como flujos gravitacionales de sedimentos de distintas granulometrías (Fig. 8a). Algunos bancos poseen clastos de más de $1 \mathrm{~m}$. En los niveles basales suelen observarse brechas caóticas integradas 


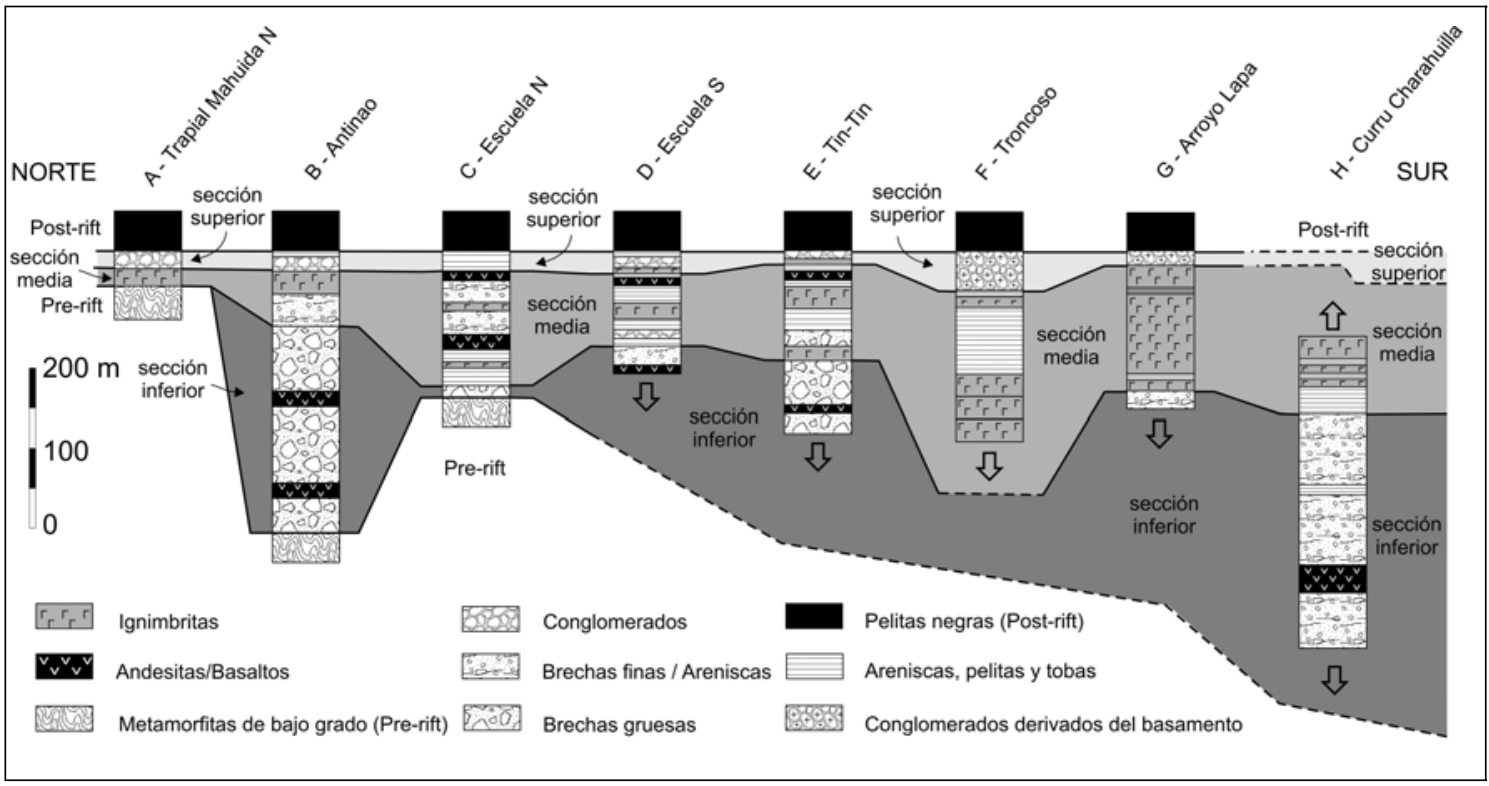

FIG. 7. Distribución de facies y espesores de las unidades de 'sin-rift' en las ocho secciones de detalle estudiadas (véase localización dentro del área de estudio en la Fig. 6).

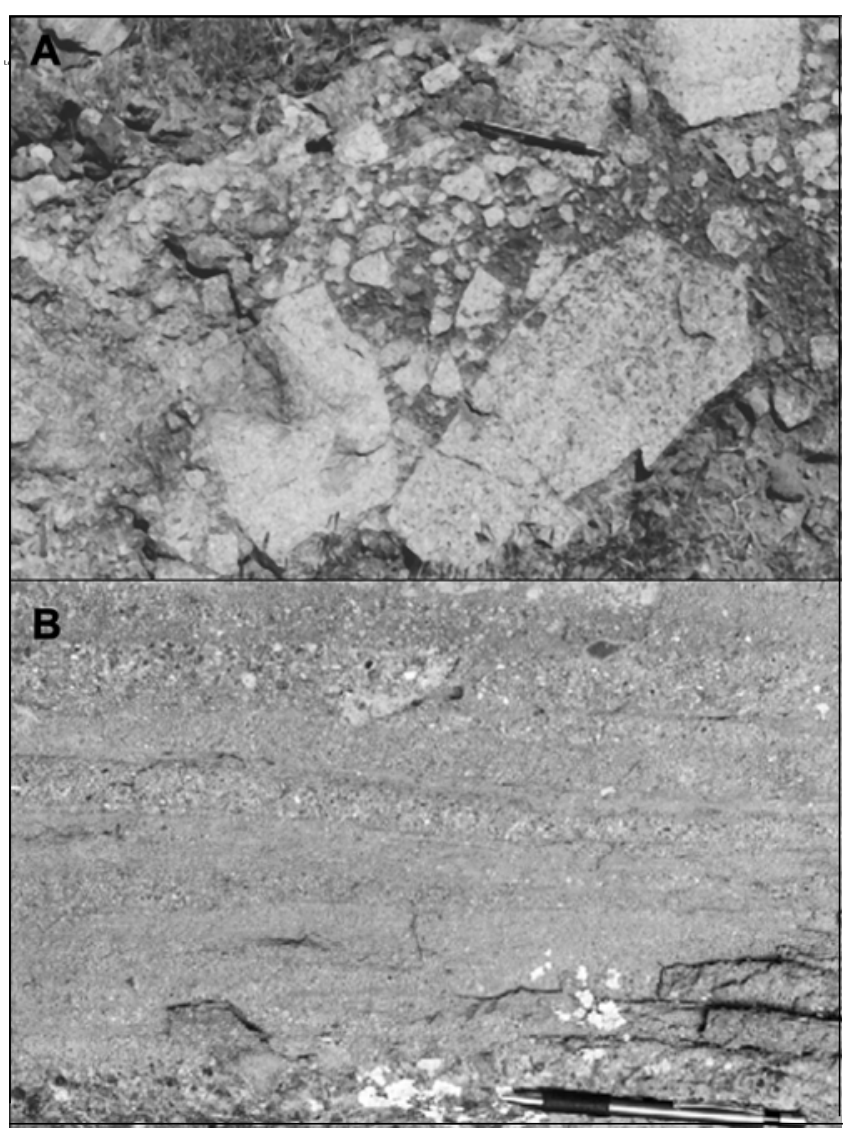

FIG. 8. La sección inferior del 'sin-rift' en la sierra de Chacaico. A. Brechas gruesas volcánicas (autobrecha). Sección Antinao. B. Facies arenosas gruesas a medianas con estratificación horizontal interpretadas como depósitos de flujos hiperconcentrados. Sección inferior del perfil Currú Charahuilla. 
por clastos exclusivamente provenientes del basamento ígneo-metamórfico, aunque la participación de material con proveniencia del 'pre-rift' se hace muy escasa hasta la parte alta de toda la secuencia de 'sin-rift'. En el flanco oriental del cerro Trapial Mahuida (sección Antinao, B; Fig. 7) dominan las brechas gruesas, masivas, matriz sostén, con estratificación grosera. Niveles de brechas finas y areniscas volcanoclásticas en láminas horizontales de escala centimétrica se intercalan con los depósitos gruesos y pueden ser interpretados como el producto de flujos fluidos hiperconcentrados (Fig. 8b).

En el área sur (Secciones Lapa y Curru Charahuilla, G, H; Fig. 7) la unidad inferior está dominada por depósitos epiclásticos bien estratificados, arenosos a brechosos finos, con pelitas intercaladas. Las areniscas y brechas son moderadamente seleccionadas, macizas, con estratificación horizontal y bases frecuentemente erosivas. Teniendo en cuenta esas características, estos depósitos pueden ser interpretados como depósitos de flujos gravitacionales e hiperconcentrados. En menor proporción aparecen conglomerados y areniscas gruesas bien seleccionados y con estratificación entrecruzada en artesa, asociados a corrientes fluidas. En la sección Curru Charahuilla ( $\mathrm{H}$; Fig. 7) afloran rocas basálticas efusivas e intrusivas someras conformando un relieve volcánico irregular sobre el que se apoyan discordantemente algunas de las secuencias clásticas señaladas.

La sección basal se apoya sobre el basamento ígneo-metamórfico en el sector norte (A; Fig. 6), aunque ese contacto pasa a ser tectónico en el borde sur del cerro Trapial Mahuida. En el extremo sur de la sierra también se observa en contacto sobre el basamento y, por falla, con secuencias más jóvenes del 'post-rift'. Otros afloramientos menores de esta sección se registran en el arroyo TinTin y en el núcleo del anticlinal en el arroyo Lapa (E, G; Fig. 6).

\section{SECCIÓN MEDIA}

En discordancia o localmente en concordancia sobre la sección basal se apoya una serie de sedimentitas clásticas y piroclásticas, con una participación dominante de rocas derivadas de flujos piroclásticos, que culminan con intercalaciones de calizas laminadas y lavas basálticas. El límite entre las secciones inferior y media de la secuencia de 'sin-rift' se ha localizado a partir del nivel en el cual deja de ser dominante la asociación de rocas volcánicas andesíticas basales y sus derivados sedimentarios y comienzan a ser importantes los depósitos piroclásticos primarios riolíticos. Esta sección varía entre 60 m y 120 m de potencia. Desde el punto de vista de la composición litológica es la más compleja de las unidades del 'sin-rift', siendo la sección más representativa la que se encuentra inmediatamente al oeste de la Escuela No. 83 (sección Escuela S, D; Figs. 6 y 7). En la base dominan los depósitos de tobas y tufitas riolíticas de tonalidades verdosas en bancos tabulares de 10 a 50 centímetros. Intercalados entre estas rocas aparecen depósitos de conglomerados gruesos y areniscas mayormente polimícticos conformando niveles lenticulares de hasta $200 \mathrm{~m}$ de ancho y 20 $\mathrm{m}$ de potencia (Fig. 9A). La característica más saliente de esta sección es el desarrollo de potentes secuencias de ignimbritas de composición riolítica que en la sección del arroyo Lapa superan ampliamente los $100 \mathrm{~m}$ de potencia. Estas ignimbritas poseen restos de troncos silicificados y carbonizados y evidencias de alteración hidrotermal (D'Elía y Franzese, 2005). En la parte superior, asociados con depósitos de tobas y tufitas verdosas y castañas, afloran bancos carbonáticos laminados, de hasta $1 \mathrm{~m}$ de potencia, que integran una secuencia de ambiente lacustre (Muravchik y Franzese, 2005) con espesores de hasta $30 \mathrm{~m}$ (Fig. 9B). Coronando la unidad intermedia, aunque con una distribución irregular, se observa un conjunto de cuerpos efusivos basálticos que en muchas localidades se apoyan sobre los niveles lacustres. Estas lavas aumentan drásticamente su potencia en el área que se encuentra inmediatamente al norte del arroyo Lapa, alcanzando hasta $100 \mathrm{~m}$.

Al norte del área de estudio, esta sección se acuña considerablemente y está construida sólo por un nivel de ignimbritas de menos de $50 \mathrm{~m}$ de potencia que se apoya directamente sobre el basamento y es cubierto por depósitos de la sección superior.

\section{SECCIÓN SUPERIOR}

La secuencia de 'sin-rift' culmina con una sección conformada por depósitos epiclásticos gruesos asociados con depósitos piroclásticos de flujo 


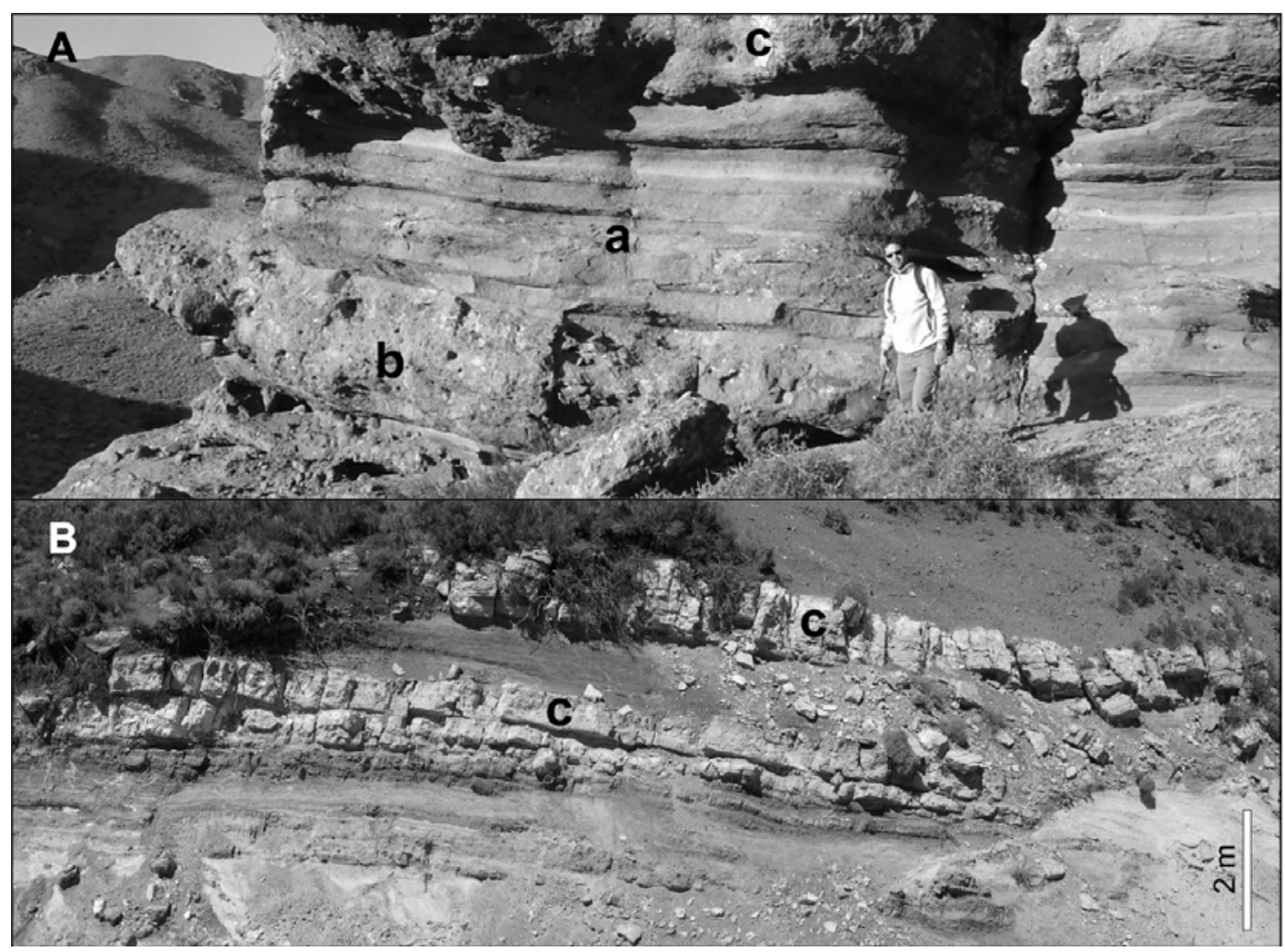

FIG. 9. La sección media del 'sin-rift' A. Depósitos clásticos intercalados entre las facies volcaniclásticas finas de la parte media de la sección Escuela S (a: areniscas gruesas a medianas, b: brechas gruesas, c: conglomerados gruesos). B. Depósitos carbonáticos (c) tabulares intercalados en una sección volcanoclástica de grano fino interpretados como depósitos lacustres. Sección media de la sección Escuela S.

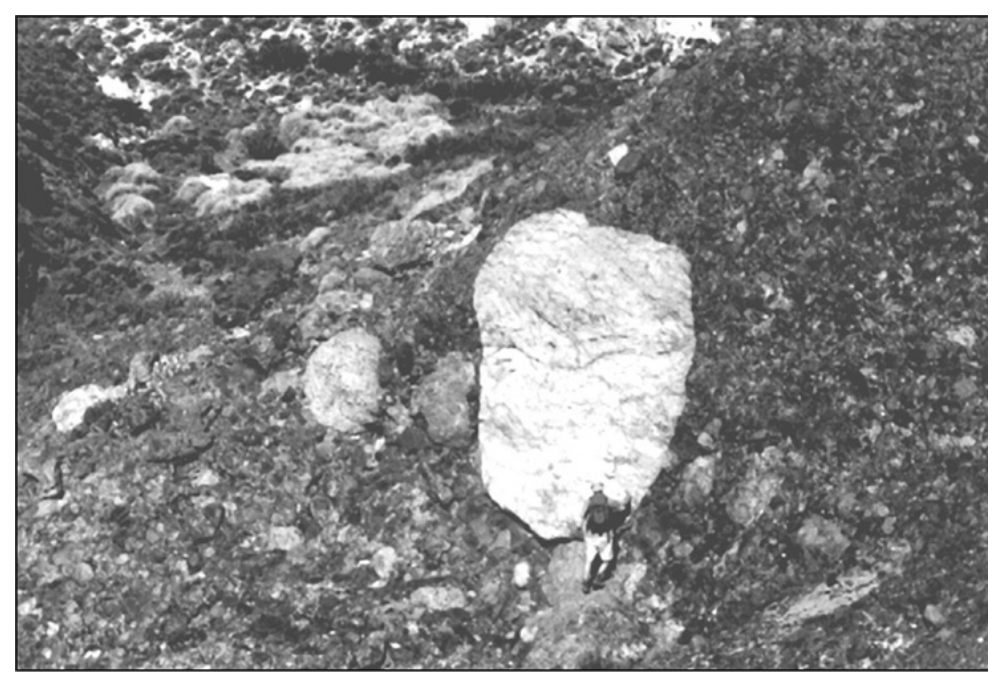

FIG. 10. Depósitos de conglomerados muy gruesos de la sección superior del 'sin-rift' en la sección Troncoso. Nótese la figura humana como escala. 
y de caída. Esta unidad es la más delgada, no alcanzando los $100 \mathrm{~m}$ de potencia. El límite inferior está marcado por la reaparición de las facies clásticas gruesas por sobre los niveles de tufitas, depósitos lacustres y lavas basálticas de la sección media. Los conglomerados y brechas son polimícticos, con clastos de hasta $30 \mathrm{~cm}$ de diámetro y base erosiva. Se han observado megaconglomerados que poseen clastos de composición granítica de hasta $5 \mathrm{~m}$ (Fig. 10). Los depósitos piroclásticos que se intercalan son delgados niveles de tobas e ignimbritas de hasta 1 metro. La sección más completa se encuentra en el norte de la sierra, en la sección Escuela S (D; Fig. 7). Allí aflora con un espesor de $35 \mathrm{~m}$ dominado por conglomerados y brechas con intercalaciones finas de ignimbritas y tobas verdosas y amarillentas. En esta sección son claramente distinguibles dos unidades de flujo piroclástico superpuestas, las cuales son cubiertas por brechas y conglomerados polimícticos, moderadamente a mal seleccionados. En la sección Troncoso (F; Fig. 7), la parte alta de la secuencia está integrada totalmente por conglomerados, alcanzando más de $40 \mathrm{~m}$ de potencia. En la sección del arroyo Lapa (G; Fig. 7) dominan los depósitos ignimbríticos, quedando las facies conglomerádicas reducidas a la porción superior de la columna.

\section{CORRELACIÓN ENTRE LAS SECCIONES}

El análisis estratigráfico de las secciones relevadas hace posible distinguir la existencia de fuertes variaciones de espesor entre las unidades consideradas así como cambios faciales y composicionales a lo largo de toda el área de estudio (Fig. 7). Los espesores de la unidad inferior son variables desde unos pocos metros hasta más de $330 \mathrm{~m}$ tal como se observa en el sector sur de la sierra (sección Curru Charahuilla, H; Fig. 7). En algunos sectores existen claras evidencias de que no fue acumulada, tal como en el margen más septentrional del área de estudio, en donde la sección media apoya directamente sobre el basamento. Algunos de estos cambios de espesor se observan en cortas distancias a partir de su acuñamiento sobre bloques de 'pre-rift' que habrían actuado como altos topográficos durante la acumulación (sección Escuela N, C; Fig. 7). Otros cambios son más bruscos, asociados probablemente a la presencia de fallas que, de esta manera, demuestran haber sido activas durante el 'sin-rift', tal como se observan en el margen sureste del cerro Trapial Mahuida (Figs. 6 y 7).

La sección intermedia muestra una variación de espesores entre $60 \mathrm{~m}$ y $120 \mathrm{~m}$ que acompaña parcialmente a las variaciones observadas en la sección inferior. Las mayores potencias se encuentran en las secciones Arroyo Lapa y Troncoso, al sur de la sierra, observándose un acuñamiento paulatino hacia el sur del cerro Trapial Mahuida. Las potentes ignimbritas de esta sección, que en el perfil del arroyo Lapa (G; Fig. 7) poseen hasta $100 \mathrm{~m}$ y forman la mayor parte del cerro Keli Mahuida, prácticamente desaparecen en la sección Escuela N. Las secuencias piroclásticas de flujo son reemplazadas allí por una asociación epiclásticapiroclástica integrada por tobas y tufitas arenosas en las cuales se intercalan secuencias conglomerádicas canalizadas de escaso desarrollo areal. Finalmente, en la parte más septentrional del área de estudio el espesor de las ignimbritas que integran la sección vuelve a ser significativo en las secciones Antinao y Trapial Mahuida N (B, A; Fig. 7).

La sección superior no muestra el mismo patrón de distribución de espesores dentro de la secuencia de 'sin-rift'. En el área central de la sierra su distribución es irregular, con fuertes variaciones de espesor en cortas distancias sin relación con los cambios observados en las secciones más antiguas (Fig. 7).

\section{DISCUSIÓN}

Los estudios realizados sobre la secuencia de 'sin-rift' permiten verificar la naturaleza volcanosedimentaria de su relleno. Tal como ha sido descrito en otras áreas de la cuenca, la secuencia se inicia con una clara tendencia volcánica con abundancia de andesitas y brechas volcánicas y evoluciona hacia depósitos ignimbríticos ácidos en la parte media y superior (Franzese et al., 2006). El estilo y magnitud del relleno de 'sin-rift' en la sierra de Chacaico es similar al relevado en otras 
áreas vinculadas con la apertura de la Cuenca Neuquina (Gulisano y Pando, 1981; Gulisano et al., 1984; Gulisano y Gutiérrez-Pleimling, 1995; Álvarez y Ramos, 1999; Pángaro et al., 2002).

La sección inferior es la que muestra la mayor variabilidad de espesor. La fuerte variación de espesores en cortas distancias observada en el norte de la sierra de Chacaico (secciones Antinao y Escuela N, por ejemplo; Fig. 7) implicaría la existencia de un fuerte control inicial del relleno con generación de estructuras angostas y compartimentalizadas tal como se registra en las fases de 'rift' inicial en otras secuencias continentales (Gawthorpe y Leeder, 2000).

La secuencia media se encuentra mucho más integrada dentro del área estudiada, aunque muestra una variación que acompaña lo observado en las brechas y volcanitas iniciales (Fig. 7). El fuerte apilamiento de unidades de flujo piroclástico entre la sección del Arroyo Lapa y el borde sur de la estructura y el espesor importante de la secuencia de 'sin-rift' en la misma área podrían ser evidencias de que ese sector habría sido el más cercano al eje depositacional de la cubeta. Los espesores decrecen hacia el norte hasta hacerse mínimos en el límite septentrional de los afloramientos (Fig. 7). En este esquema la Falla de Las Coloradas (Figs. 5 y 6) habría actuado como borde activo principal del depocentro.

La existencia de importantes variaciones en potencia y espesor de las unidades de 'sin-rift' en sentido norte-sur se pueden analizar en el contexto estructural de la sierra de Chacaico, ya que parecen acompañar la distribución de las culminaciones oblicuas y transversales a la orientación general de la sierra. Los cambios estratigráficos registrados encuentran un gran correlato con la disposición de las estructuras antiformes del cerro Trapial Mahuida, Keli Mahuida y Curru Charahuilla, así como con los límites este-oeste de toda la sierra (Figs. 3, 5 y 6). Las variaciones de espesores de las secciones inferior y media se encuentran íntimamente asociadas a la distribución de los anticlinales oblicuos ya señalados. Cada una de esas antiformas muestra la presencia de rocas de niveles estratigráficos más antiguos en posiciones estructuralmente más elevadas que las de su entorno. En el cerro Trapial Mahuida (Figs. 3 y 6 ), el basamento y la sección inferior de la secuencia de 'sin-rift' se hallan a 1.750 m de altura. La región

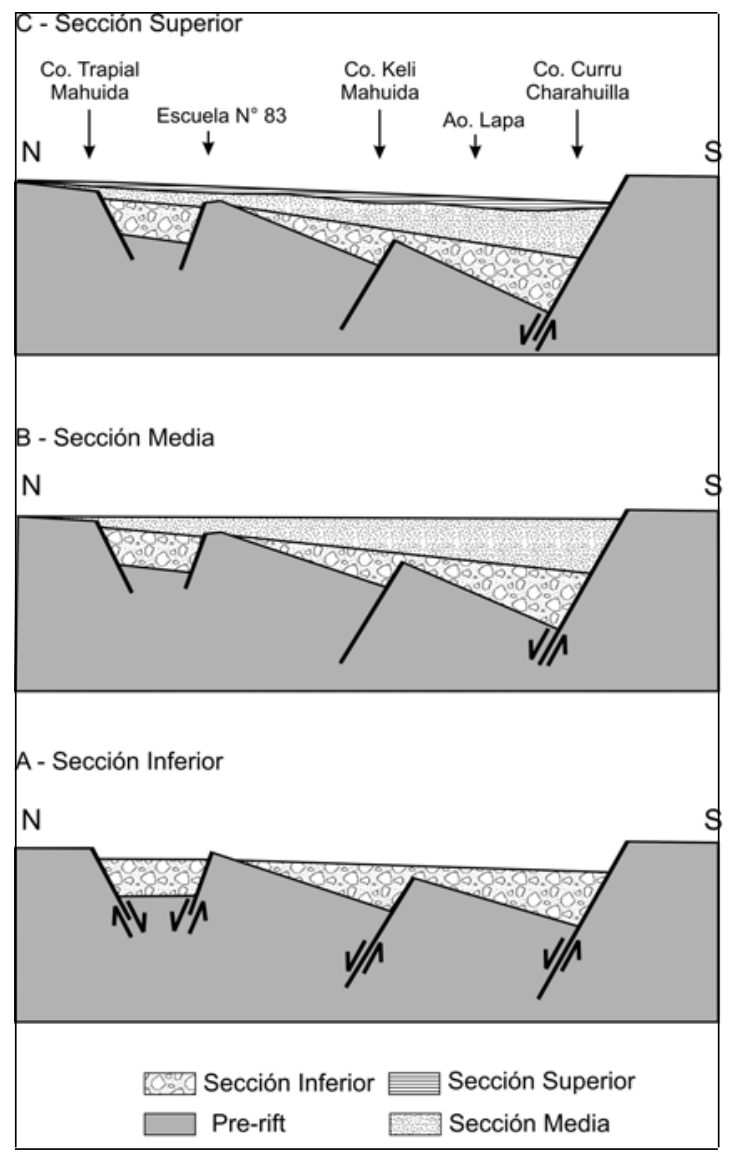

FIG. 11. Modelo general de evolución del relleno de 'sin-rift' en la sierra de Chacaico

inmediatamente al sur muestra el afloramiento de las secuencias medias y altas de la secuencia en posición estructuralmente más baja, inferior a los $1.600 \mathrm{~m}$. En el cerro Keli Mahuida, las rocas ignimbríticas de la sección media se alzan fuertemente, levantándose entre 200 m y 300 m por sobre las secuencias de la sección media y superior que afloran al norte y al sur del anticlinal oblicuo (Figs. 3 y 5). Finalmente, en el área del cerro Curru Charahuilla las unidades de la sección basal están elevadas por sobre terrenos cretácicos aflorando al sur de la Falla de las Coloradas (Figs. 5 y 6$)$.

Los afloramientos de la secuencia de 'sin-rift' afloran en toda la cuenca, esencialmente por la inversión tectónica acaecida entre el Jurásico y el Cenozoico (Vergani et al., 1995), apareciendo asociadas a grandes anticlinales y bloques 
levantados por la inversión estructural de antiguas fallas extensionales activas durante la etapa inicial de la cuenca (Zapata et al., 1999). La integración de la información estratigráfica y estructural en la sierra de Chacaico permite suponer que las estructuras anticlinales oblicuas serían, por lo tanto, plegamientos asociados a la inversión de fallas extensionales originales. Las variaciones nortesur registradas en litofacies y espesores de las unidades internas de 'sin-rift' responden directamente a una disposición noreste a esteoeste en el rumbo de las fallas normales del depocentro (Fig. 11). Los máximos espesores registrados en el sur indican que la Falla de las Coloradas podría haber actuado como límite principal de la cubeta, mientras que el escaso a nulo espesor presente en el borde norte marcaría el otro límite de depositación de la secuencia, ambos con la misma orientación este-oeste. En el interior del depocentro, los pisos de los bloques internos oblicuos, sobreelevados y rotados, habrían generado dorsales de rumbo noreste y limitado cubetas aisladas durante el relleno de la sección inferior (Fig. 11). Durante la evolución estructural posterior las áreas de depositación se habrían integrado paulatinamente, aunque los máximos espesores se mantuvieron asociados al borde activo de la Falla de Las Coloradas (Fig. 11). La sección superior se habría acumulado bajo condiciones de menor acomodación, en un depocentro ya completamente integrado.

La disposición estructural observada en la sierra de Chacaico muestra un patrón similar al definido para la megaestructura de inversión de la dorsal de Huincul, registrada en el subsuelo inmediatamente al ENE del área de estudio (Fig. 1). La disposición de los rasgos estructurales de la dorsal está definida por la existencia de una faja de pliegues y fallas subparalelos, de orientación este-oeste a noreste, que cortan la Cuenca Neuquina en dos áreas (Orchuela et al., 1981; Vergani, 2005). Los segmentos deformados representan la inversión de fallas normales principales que habrían controlado la formación de depocentros iniciales y registran los máximos espesores de 'sin-rift' (Vergani, 2005). Teniendo en cuenta estas similitudes se verifica que la zona de la sierra de Chacaico formaría parte integral de la dorsal de Huincul, compartiendo sus principales rasgos morfoestructurales y estratigráficos. La disposición de esas fracturas iniciales habría sido oblicua a la conformación actual de la sierra. La disposición norte-sur del anticlinal de Chacaico, rasgo que no es común en la dorsal de Huincul, es subparalela a otras estructuras de deformación neógena en el frente orogénico andino y se habría superpuesto sobre las estructuras este-oeste más antiguas oscureciendo las relaciones estructurales del 'sin-rift'.

\section{CONCLUSIONES}

La secuencia de 'sin-rift' de la sierra de Chacaico evolucionó en un ambiente volcánico continental controlado por estructuras dispuestas este-oeste y noreste durante el Triásico Superior-Jurásico Inferior.

La secuencia mayor puede ser subdividida en tres partes: la sección inferior, compuesta por volcanitas andesítico-basálticas y sus depósitos de retrabajo sedimentario, muestra fuertes variaciones de espesor al haberse depositado en cubetas aisladas. En la sección intermedia dominan las rocas de origen piroclástico y composición riolítica, mientras que la sección superior se caracteriza por la asociación entre depósitos brechosos y piroclásticos primarios. La distribución de estas últimas secciones está más integrada dentro de toda la sierra que la unidad basal.

Los máximos espesores del relleno se encuentran en el sur, en asociación con la presencia de un límite estructural abrupto conformado por la Falla de Las Coloradas, que actuó como límite activo principal del depocentro. El límite norte también está marcado por la existencia de una falla importante de orientación este-oeste.

El esquema tectonoestratigráfico general es coincidente con la evolución de la dorsal de Huincul, rasgo de inversión de depocentros estructurales más prominente del área sur de la Cuenca Neuquina. 


\section{AGRADECIMIENTOS}

Este trabajo fue financiado por fondos provenientes de los proyectos PICT 07-8451 у PICT 25304 de la Agencia Nacional de promoción Científica y Tecnológica (ANPCYT; Argentina), 11/N385 de la Universidad Nacional de La Plata (UNLP; Argentina) e YPF S.A. Se agradece la ayuda y hospitalidad proporcionada por el personal de la Escuela No.
83 y toda la comunidad de Chacaico Sur, Neuquén. Los autores agradecen a los revisores $\mathrm{H}$. Leanza (CONICET-UNLP), V. Ramos (CONICET-UBA) y E. Rossello (CONICET-UBA) y al editor de la Revista Geológica de Chile, M. Suárez, por sus valiosas sugerencias.

\section{REFERENCIAS}

Álvarez, P.P.; Ramos, V.A. 1999. The Mercedario rift system in the principal Cordillera of Argentina and Chile ( $\left.32^{\circ} \mathrm{LS}\right)$. Journal of South American Earth Sciences 12: 17-31.

D’Elía, L.; Franzese, J.R. 2005. Caracterización litológica y estructural de ignimbritas precuyanas en la sierra de Chacaico, Neuquén, con énfasis en su potencial petrolero. In Congreso de exploración de hidrocarburos No. 6. Trabajos técnicos, Reservorios y desarrollo de reservas. Resúmenes de trabajos: 27. Edición en CD-ROM. Mar del Plata, Argentina.

Franzese, J.R. 1995. El Complejo Piedra Santa (Neuquén, Argentina): Parte de un cinturón metamórfico de edad neopaleozoica del Gondwana suroccidental. Revista Geológica de Chile 22: 193-202.

Franzese, J.R; Spalletti, L.A. 2001. Late Triassic-Early Jurassic continental extension in southwestern Gondwana: tectonic segmentation and pre-break-up rifting. Journal of South American Earth Sciences 14: 257-270.

Franzese, J.R.; Veiga, G.D.; Schwarz, E.; Gómez-Pérez, I. 2006. Tectono-stratigraphic evolution of a Mesozoic Graben Border System: the Chachil depocentre, southern Neuquén Basin, Argentina. Journal of the Geological Society of London 163: 207-221.

Gawthorpe, R.L.; Leeder, M.R. 2000. Tectonosedimentary evolution of active extensional basins. Basin Research 12: 195-218.

Gulisano, C.A.; Gutiérrez-Pleimling, A.R. 1995. The Jurassic of the Neuquen Basin, a) Neuquen Province. In International Congress on Jurassic Stratigraphy and Geology No. 4, Field guide, Serie E, Asociación Geológica Argentina: 111 p. Buenos Aires.

Gulisano, C.A.; Pando, G.A. 1981. Estratigrafía y facies de los depósitos jurásicos entre Piedra del Aguila y Sanico, Departamento Collon Cura, Provincia del Neuquén. In Congreso Geológico Argentino, No. 8 , Actas 3: 553-577. San Luis.

Gulisano, C.A.; Gutiérrez-Pleimling, A.R.; Digregorio, R.E. 1984. Esquema estratigráfico de la secuencia jurásica del oeste de la provincia de Neuquén. In Congreso Geológico Argentino No. 9. Actas 1: 236259. San Carlos de Bariloche.

Lambert, L.R. 1946. Contribución al conocimiento de la Sierra de Chacay-Có (Neuquén). Revista de la Asociación Geológica Argentina 1 (4): 231-256.

Leanza, H. 1990. Estratigrafía del Paleozoico y Mesozoico anterior a los movimientos intermálmicos en la comarca del Cerro Chachil, Provincia del Neuquén. Revista de la Asociación Geológica Argentina 45 (34): $272-299$.

Leanza, H.; Llambías, E.J.; Carbone, O. 2005. Unidades estratigráficas limitadas por discordancias en los depocentros de la cordillera del Viento y la sierra de Chacaico durante los inicios de la cuenca Neuquina. In Congreso de exploración de hidrocarburos, No. 6. Trabajos técnicos. Reservorios y desarrollo de reservas. Resúmenes de trabajos: 53. Edición en CDROM. Mar del Plata.

Legarreta, L.; Uliana, M.A. 1996. The Jurassic succession in west-central Argentina: stratal pattern, sequences and paleogeographic evolution. Palaeogeography, Palaeoclimatology \& Palaeoecology, 120: 303-330.

Muravchik, M.; Franzese, J.R. 2005. Carbonatos lacustres someros en las facies volcaniclásticas del Precuyano de la sierra de Chacaico, Neuquén. In Congreso Geológico Argentino No. 16, Actas 3: 111116. La Plata.

Orchuela, I.A.; Ploszkiewicz, J.V.; Viñes, R. 1981. Reinterpretación estructural de la denominada 'Dorsal Neuquina'. In Congreso Geológico Argentino, No. 8. Actas 3: 281-293. San Luis.

Pángaro, F.; Corbera, R.; Carbone, O.; Hinterwimmer, G. 2002. Los reservorios del Precuyano. In Rocas Reservorio (Schiuma, M.; Hinterwimmer, G.; Vergani, G., editores). Congreso de exploración y desarrollo de hidrocarburos, No. 5, Actas: 229-254. Mar del Plata.

Spalletti, L.A.; Arrondo, O.G.; Morel, E.M.; Ganuza, D.G. 1991. Evidencias sobre la edad Triásica de la Forma- 
ción Lapa en la región de Chacaico, Provincia del Neuquén. Revista de la Asociación Geológica Argentina 46 (3-4): 167-172.

Suero, T., 1951. Descripción geológica de la Hoja 36c, Cerro Lotena (Neuquén). Dirección Nacional de Minería, Boletín 76: 67 p. Buenos Aires.

Vergani, G.D. 2005. Control estructural de la sedimentación jurásica (Grupo Cuyo) en la Dorsal de Huincul, cuenca Neuquina, Argentina. Modelo de falla lístrica rampa-plano, invertida. Boletín de Informaciones Petroleras 1: 32-42. Buenos Aires.

Vergani, G.D.; Tankard, A.J.; Belotti, H.J.; Welsink, H.J. 1995. Tectonic evolution and paleogeography of the Neuquen Basin, Argentina. In Petroleum Basins of South America (Tankard, A.J.; Suarez Soruco, R.;
Welsink, H.J.; editors). American Association of Petroleum Geologists. Memoirs 62: 383-402.

Volkheimer, W. 1973. Palinología estratigráfica del Jurásico de la Sierra de Chacai Co y adyacencias (cuenca Neuquina, Republica Argentina). 1Estratigrafía de las formaciones Sierra Chacai Co (Pliensbachiano), de Los Molles (Toarciano, Aaleniano), Cura Niyeu (Bayociano) y Lajas (Caloviano inferior). Revista de la Asociación Geológica Argentina 10: 105-129.

Zapata, T. R.; Brissón, I.; Dzelalija, F. 1999. La estructura de la faja plegada y corrida andina en relación con el control del basamento de la cuenca Neuquina. Boletín de Informaciones Petroleras 60 (2): 112-121. Buenos Aires. 